\title{
Systèmes Agroforestiers A Garcinia Kola Heckel Au Sud-Est Du Bénin : Distribution Géographique, Connaissances Endogènes Et Retombées Financières
}

\author{
Sylvie Codjia, \\ Augustin Aoudji, \\ Kourouma Koura, \\ Jean Cossi Ganglo,
}

Laboratoire des Sciences Forestières, Ecole d'Aménagement et Gestion de

l'Environnement, Faculté des Sciences Agronomiques,

Université d'Abomey Calavi

Doi: 10.19044/esj.2018.v14n12p188 URL:http://dx.doi.org/10.19044/esj.2018.v14n12p188

\begin{abstract}
This study was carried out in three districts (Avrankou, Adjarra, and Ifangni) of south-eastern Benin, and aimed to analyze the socio-economic and ethnobotanical importance of Garcinia kola Heckel, in order to contribute to their sustainable management. Focus groups were conducted with three groups, composed of 10 owners of $G$. kola per district each and the result showed that Garcinia kola Heckel became extinct in their naturally occurring state in Benin. Besides, a survey was conducted among 140 owners of G. kola randomly selected in the study area. The results showed that the average number of G. kola feet per owner varies between 3 and 4, and the annual income generated from seeds marketing by an owner is XOF 1036000 in Adjarra district, XOF 356250 in Avrankou district, and XOF 290892 in Ifangni district. Furthermore, the ethnobotanical study showed that $G$. kola organs are well known and used by local people for medicine, food (for both humans and animals), commercial, spiritual purpose, and for timber and shading. The medicinal use was the most widespread with a diversity of uses (UD $=0.64$ in Adjarra, UD $=0.61$ in Avrankou and UD $=0.68$ in Ifangni). The old men held more knowledge about the use of various organs of the species (ID $=0.91$, IE $=0.91)$, while young women hold less knowledge $\quad(\mathrm{ID}=0.20, \mathrm{IE}=0.20)$. The seeds are the most common part used regardless of the district considered.
\end{abstract}

Keywords: Garcinia kola, geographical distribution, endogenous knowledge, financial income, South-eastern Benin 


\section{Résumé}

La présente étude a été conduite dans les communes d'Avrankou, d'Adjarra et d'Ifangni au sud-est du Bénin. L’objectif a été d'analyser l'importance socio-économique et ethnobotanique des populations de Garcinia kola Heckel, afin de contribuer à leur gestion durable. Des entretiens ont été réalisés avec trois groupes de 10 propriétaires par commune et ont permis de montrer que $G$. kola est éteinte à l'état naturel au Bénin. Une enquête a été réalisée auprès de 140 propriétaires de pieds de G. kola. Les résultats ont montré que le nombre moyen de pieds de $G$. kola varie entre 3 et 4 pieds/propriétaire. La vente des graines génère des revenus, à raison de $1036000 \mathrm{FCFA} /$ propriétaire/an à Adjarra, $356250 \mathrm{FCFA} /$ propriétaire/an à Avrankou et 290892 FCFA/propriétaire/an à Ifangni. L'étude ethnobotanique a montré que l'espèce est très connue et utilisée par les populations locales qui ont des connaissances relatives aux usages médicinaux, alimentaires (pour les hommes et les animaux), commerciaux, ombrager, bois de feux et médicomagique. L'usage médicinal est plus exploité avec une valeur de diversité des usages $(\mathrm{UD}=0,64$ à Adjarra, $\mathrm{UD}=0,61$ à Avrankou et $\quad \mathrm{UD}=0,68$ à Ifangni). Les hommes âgés de plus de 40 ans détiennent plus de connaissances sur l'utilisation de l'espèce (ID $=0,91$; IE $=0,91$ ) tandis que les jeunes femmes détiennent moins de connaissances sur l'espèce (ID $=0,20$; $\mathrm{IE}=0,20)$. Les graines constituent l'organe le plus utilisé quelle que soit la commune considérée.

Mots clés : Garcinia kola, distribution géographique, connaissances endogènes, retombées financières, Sud-Est du Bénin

\section{Introduction}

Les ressources forestières du Bénin sont assez limitées. Selon la FAO (2011), les forêts du Bénin couvrent une superficie de 4.561.000 ha. Ces maigres ressources sont en proie à une dégradation alarmante (Awokou et al., 2009). D’après les derniers chiffres de la FAO (2011), le Bénin perd en moyenne 50000 ha de forêts chaque année. Aux pertes de superficies forestières correspondent des pertes proportionnelles en espèces (Mc Arthur, 1967). Or la majorité de ces espèces fournissent aux populations rurales des produits indispensables à leur survie. Kabuye (2000) souligne à cet effet que les habitants des forêts, ainsi que les communautés vivant à proximité et dépendant de celles-ci pour leur subsistance, ont une connaissance empirique très riche sur les espèces génératrices de Produits Forestiers Non Ligneux (PFNL), notamment les plantes sauvages. Ces essences sont utilisées dans les domaines tels que la médecine et l'alimentation. Elles fournissent des légumes, fruits, noix, huiles, résines, fibres, fourrage, tisanes, bois de service 
et bois d'œuvre (Ambé, 2001, Koura et al., 2011) et permettent de générer des emplois et des revenus aux populations.

Garcinia kola Heckel (Clusiaceae) fait partie de ces espèces génératrices de PFNL. C'est un PFNL à importance socio-économique au Nigéria (Yakubu et al., 2014). Elle est reconnue pour ses utilités aux plans économique, alimentaire, nutritionnel, sanitaire, social, culturel, cultuel, cosmétique, pharmaceutique, etc. (Akoègninou et al., 2006 ; Aké et al., 2013). C'est un arbre qui a été désigné comme une "plante miracle " parce que presque toutes ses parties ont été jugées d'une importance médicinale avérée (Oguntola, 2008). C'est l'une des premières plantes médicinales du continent africain (Han et al., 2005). En Afrique de l'Ouest, son importance socioéconomique est élevée (Han et al., 2005). La graine, riche en biflavonoïde, xanthones, alcaloïdes, benzophénone (Anegbeh et al., 2006) est un masticatoire utilisé dans les cérémonies culturelles et sociales (Farombi et al., 2000). Séchées et broyées, ces graines sont mélangées à du miel pour produire un sirop traditionnel contre la toux (Onunkwo et al., 2004). Les sous-produits de cet arbre sont également utiles. Son bois fait un excellent bois de chauffage. Sa cime arrondie dense en fait un arbre idéal pour l'ombre près des habitations (Taiwo et al., 1999). Ses tiges et rameaux sont utilisés comme brosses à dents dans plusieurs régions africaines (Tchoundieu et al., 2000). Ils ont été commercialisés dans les grandes villes pendant des années, offrant des soins dentaires naturels à cause de leur goût amer et des activités antibactériennes de leurs extraits (Agyili et al., 2006).

G. kola est exposé à de graves menaces qui pèsent sur sa conservation, de par la dégradation de l'environnement, la surexploitation et l'absence de méthodes de gestion de l'essence (CTA, 2007). Elle est potentiellement menacée par les prélèvements de l'écorce effectués par les villageois pour contribuer à la fermentation du vin de palme (CTA, 2007). Elle est même inscrite sur la liste rouge de l'UICN (Neuenschwander et al., 2011).

Très peu de recherches ont été menées sur G. kola au Bénin. C'est une espèce de la famille des Clusiacées, originaire de la région tropicale précisément de l'Afrique. Elle se développe dans les forêts denses et les forêts semi-décidues. Au Bénin, le Sud du pays représente sa zone de prédilection naturelle et les peuplements les plus importants se retrouvent dans les départements de l'Ouémé et du Plateau (Adjanohoun et al., 1989). Selon Adjanohoun et al. (1989), il existerait 13 espèces du genre Garcinia en Afrique de l'Ouest dont quatre rapportées dans la flore du Bénin (Akouègninou et al., 2006). G. kola représente l'espèce prioritaire pour la conservation suivant les critères de répartition, de multiplication, d'utilisations potentielles et d'échanges régionaux (Eyog Matig et al., 2000). Kouchadé (2013) a évalué les connaissances endogènes et écophénotypiques de G. kola au sud-Bénin. Etant donné que la graine de l'espèce est prisée au Bénin, il faudra une étude socio- 
économique couplée à une étude ethnobotanique afin de vérifier le potentiel économique lié aux usages faits des différentes parties de cette espèce. Aussi, la distribution géographique étant la base fondamentale de l'étude d'une espèce, il urge que les différentes zones dans lesquelles l'espèce se retrouve dans notre milieu d'étude soient connues. Alors, l'objectif principal de la présente étude est de déterminer la distribution géographique ainsi que les importances socio-économique et ethnobotanique des populations de G. kola au sud-est du Bénin afin de proposer des solutions pour leur gestion durable.

\section{Méthodologie}

\section{Milieu d'étude}

La présente étude a été faite dans les communes d'Avrankou, Adjarra et Ifangni au sud-est du Bénin (Figure 1). Ces communes ont été choisies suite à une enquête préliminaire qui a permis d'identifier les zones dans lesquelles G. kola est le plus abondant. Ces communes appartiennent à la zone guinéocongolaise et jouissent d'un climat subéquatorial avec une humidité relative de l'air de $75 \%$. La température moyenne du milieu d'étude varie entre $21,9{ }^{\circ} \mathrm{C}$ et $32,8{ }^{\circ} \mathrm{C}$ avec une pluviométrie de $1200 \mathrm{~mm}$. Dans ces trois communes, le commerce, favorisé par le voisinage du Nigéria est la principale activité de la population. Viennent ensuite l'agriculture et l'élevage, et l'industrie manufacturière avec une ampleur variable d'une commune à l'autre.

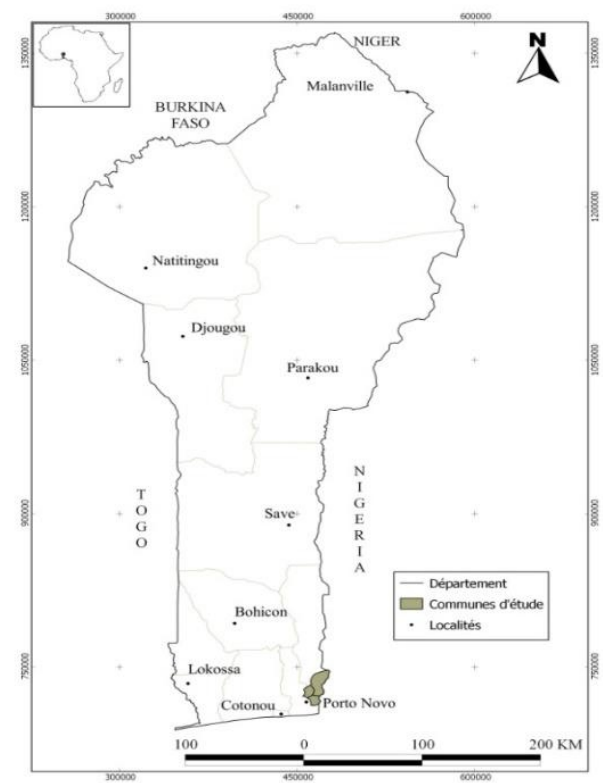

Figure 1 : Situation géographique des communes d'étude au sud-est du Bénin. 


\section{Matériel végétatif}

Garcinia kola Heckel est communément appelé en fon « ahowétin » et en yoruba « igui orogbo ». Il est un grand arbre à empâtement à la base, atteignant 35 - $40 \mathrm{~m}$ de haut (Akerele et al., 2007). Les feuilles caduques persistantes sont opposées. Les fleurs mâles possèdent quatre (4) sépales inégaux, tomenteux extérieurement, glabres intérieurement (Sibanda, 2007). Elles donnent des fruits jaunâtres à rougeâtres lisses qui sont des drupes d'environ 5-10 cm de diamètre chacune (Agyili et al., 2006) dans laquelle sont intégrées 1-4 graines brunes résineuses (Akoègninou et al., 2006). Le tronc est lisse et grisâtre à base cylindrique ou presque. L'arbre possède une racine pivotante bien ancrée dans le sol.

\section{Méthodes}

\section{Identification des zones de distribution géographique de l'espèce}

La présente étude a été faite dans les trois communes (Adjarra, Avrankou et Ifangni) du sud-est du Bénin où G. kola est présente en plantation. Le choix de ces localités s'est basé sur deux critères. Le premier critère est l'importance de l'espèce pour les propriétaires. Cette importance a été mesurée dans chaque localité (durant l'enquête exploratoire) par la présence de G. kola dans les jardins de cases, mais surtout par l'utilisation de l'espèce ou de ses produits par les populations locales. Le deuxième critère de choix des localités d'étude était relatif à l'existence de marchés où les organes de l'espèce sont commercialisés, ceci pour pouvoir avoir une idée satisfaisante de l'importance économique. L'existence de tels types de marchés dans les localités a été révélée durant les enquêtes exploratoires, sur la base d'observations directes, lorsque la prospection a lieu pendant la période de disponibilité des fruits sur le marché et, dans le cas contraire, après des enquêtes auprès des populations locales.

Pendant la phase exploratoire, les objectifs de l'étude ont été présentés aux chefs de quartier/village ainsi qu'aux acteurs du monde agricole et forestier (Institut National des Recherches Agricoles du Bénin, services forestiers, etc.) de ces différentes localités. Dans un premier temps, les populations ont été questionnées sur la localisation de l'espèce. Le "Global Positioning System" (GPS) a été utilisé, afin de matérialiser les coordonnées géographiques des populations de l'espèce identifiées. Les coordonnées enregistrées à partir du GPS ont servi à la réalisation de la carte de répartition de l'espèce dans les différentes zones d'investigation. Les données saisies dans le tableur Excel ont été importées dans le logiciel Quantum Gis version 2.6, pour réaliser la carte de répartition de $G$. kola dans les différentes zones d'investigation. 


\section{Reconstitution de l'historique des systèmes agroforestiers à Garcinia kola}

Dans les trois localités (Adjarra, Avrankou et Ifangni), un recensement de tous les paysans propriétaires de pieds de G. kola a été fait. Pour reconstituer l'historique des systèmes agroforestiers à G. kola, des techniques d'entretiens de groupe ont été utilisées. Trois groupes de 10 propriétaires par commune ont été interrogés.

Les entretiens de groupe ont permis de retracer tous les événements qui ont contribué à la disparition de l'espèce en milieu naturel et au développement des plantations. Chaque groupe était composé de dix personnes volontaires qui possèdent chacun au moins un pied de G. kola. Une discussion ouverte a été menée avec chaque groupe, sur la base d'une fiche d'enquête qui a abordé les points suivants :

- $\quad$ les formations végétales dans lesquelles G. kola est rencontré ;

- $\quad$ la persistance ou non des pieds naturels de $G$. kola dans les formations végétales ;

- $\quad$ les modes de multiplication de G. kola dans la zone d'étude ;

- $\quad$ l'année de démarrage de la plantation de $G$. kola dans la zone d'étude

;

- $\quad$ les facteurs qui sous-tendent la disparition des peuplements naturels de G. kola ;

- $\quad$ les raisons de la plantation ou non de l'espèce dans la localité.

En dehors des entretiens de groupe, des entretiens individuels ont été faits avec les propriétaires de pieds de G. kola sur la base d'un questionnaire d'enquête. Les propriétaires enquêtés sont répartis comme suit : 46 à Adjarra, 30 à Ifangni et 64 à Avrankou compte tenu de l'abondance de l'espèce dans chaque commune. Les raisons qui sous-tendent la plantation ou non de l'espèce ont été recensées de même que le nombre de pieds par propriétaire. Au cours des entretiens individuels, chaque propriétaire de pieds de G. kola a estimé les années de plantation des pieds qui lui appartiennent.

\section{Retombées financières générées par les graines de G. kola}

Le nombre moyen de pieds par propriétaire a été calculé par commune. Chaque propriétaire a estimé sa recette annuelle par pied d'arbre en fonction du nombre de bassines qu'il vend par pied.

La moyenne des recettes financières obtenues par pied par commune a été donc calculée dans le tableur Excel par la formule :

$\mathrm{Y}=\frac{1}{n} \sum_{i=1}^{n} Y i$; Avec yi représentant la recette par pied d'arbre

Pour ces données (moyenne des recettes financières obtenues par pied par commune, nombre moyen de pieds), les communes ont été comparées entre elles, grâce à une analyse de variance suivie du test de Student Newman Keuls au seuil de $5 \%$ dans le logiciel Statistica. 


\section{Caractérisation des savoirs des populations locales et leurs relations avec G. kola}

Un sondage a été effectué auprès de 140 propriétaires d'au moins un pied de G. kola, choisis de façon aléatoire dans la zone d'étude. Trente (30) propriétaires ont été enquêtés dans la commune d'Ifangni, quarante-six (46) dans la commune d'Adjarra et soixante-quatre (64) dans la commune d'Avrankou sur la base d'un questionnaire d'enquête. La taille de l'échantillon varie d'une commune à l'autre compte tenu de l'abondance de l'espèce dans chaque commune. De plus, dans certaines communes, certains propriétaires étaient réticents à répondre aux questions, ce qui a fait qu'ils n'ont pas été finalement pris en compte. Tous les enquêtés appartiennent au groupe ethnique «Goun », groupe socio-linguistique majoritaire de la zone d'étude.

Les catégories d'usage de l'espèce, les organes de l'espèce ainsi que les formes d'utilisation des organes de l'espèce ont été recensées auprès des enquêtés.

Pour la caractérisation ethnobotanique de G. kola, les indices ci-après ont été utilisés pour l'analyse des données ethnobotaniques : (1) l'indice de diversité des interviewés (ID), (2) l'indice d'équitabilité des interviewés (IE), (3) la valeur de la diversité des usages (UD), (4) la valeur d'équitabilité des usages (UE) et (5) la valeur consensuelle pour les parties de plantes (CPP). Le tableau 1 ci-dessous présente les détails explicatifs de chaque indice. Ces paramètres indiquent comment l'espèce est utilisée et comment la connaissance de ces usages est distribuée au sein des interviewés. Pour calculer la valeur de la diversité des usages (UD), des usages spécifiques ont été classées en différentes catégories. La normalité et l'homogénéité des indices ID et IE calculés ont été vérifiées et le test non paramétrique de Kruskal-Wallis, effectué avec le logiciel Minitab, a été réalisé pour faire ressortir les différences significatives dues à l'âge et au sexe. La structuration, proposée par Monteiro et al. (2006), Koura et al. (2011), suppose que les jeunes sont les personnes ayant un âge inférieur à 40 ans tandis que les personnes qui ont 40 ans et plus sont considérées pratiquement comme des vieux. Les valeurs consensuelles pour les parties de plantes (CPP) calculées par commue ont été sujettes à une Analyse en Composantes Principales (ACP) avec le logiciel $\mathrm{R}$ pour une meilleure description des relations entre les valeurs d'usage et les groupes socio-linguistiques. 
Tableau 1 : Paramètres ethnobotaniques calculés.

\begin{tabular}{|c|c|c|}
\hline Paramètres & Formules & Interprétation \\
\hline $\begin{array}{l}\text { Indice de diversité des } \\
\text { enquêtés (ID) } \\
\text { ID }=\mathrm{Ux} / \mathrm{Ut}\end{array}$ & $\begin{array}{c}\text { C'est le nombre d'utilisations } \\
\text { citées par un enquêté (Ux) divisé } \\
\text { par le nombre total d'utilisations } \\
\text { (Ut) }\end{array}$ & $\begin{array}{l}\text { Mesure combien d'enquêtés } \\
\text { utilisent l'espèce et comment } \\
\text { cette connaissance est répartie } \\
\text { entre les enquêtés }\end{array}$ \\
\hline $\begin{array}{l}\text { Indice d'Equitabilité des } \\
\text { enquêtés (IE) } \\
\text { IE = ID/IDmax }\end{array}$ & $\begin{array}{l}\text { C'est l'indice de diversité des } \\
\text { enquêtés (ID) divisé par la valeur } \\
\text { maximale de cet indice (IDmax) }\end{array}$ & $\begin{array}{l}\text { Mesure le degré d'homogénéité } \\
\text { des connaissances des enquêtés }\end{array}$ \\
\hline $\begin{array}{c}\text { Valeur de la diversité des } \\
\text { usages (UD) } \\
\text { UD = Ucx/Uct }\end{array}$ & $\begin{array}{l}\text { C'est le nombre d'usages par } \\
\text { catégorie (Ucx) divisé par le } \\
\text { nombre total d'usages pour toutes } \\
\text { les catégories (Uct) }\end{array}$ & $\begin{array}{l}\text { Mesure l'importance de chaque } \\
\text { catégorie d'utilisation et } \\
\text { comment elle contribue à la } \\
\text { valeur totale des usages }\end{array}$ \\
\hline $\begin{array}{c}\text { Valeur d'Equitabilité des } \\
\text { usages (UE) } \\
\text { UE }=\text { UD/UDmax }\end{array}$ & $\begin{array}{l}\text { C'est la valeur de la diversité des } \\
\text { usages (UD) divisée par sa valeur } \\
\text { maximale (UDmax) }\end{array}$ & $\begin{array}{l}\text { Mesure le degré d'homogénéité } \\
\text { de connaissance par rapport aux } \\
\text { catégories d'utilisations }\end{array}$ \\
\hline $\begin{array}{l}\text { Valeur consensuelle } \\
\text { pour les parties de } \\
\text { plante }(\mathrm{CPP}) \\
\mathrm{CPP}=\mathrm{Px} / \mathrm{Pt} \\
\end{array}$ & $\begin{array}{l}\text { C'est le nombre de fois où la partie } \\
\text { d'une plante est citée (Px) divisé } \\
\text { par le nombre total de fois que } \\
\text { toutes les parties sont citées }(\mathrm{Pt})\end{array}$ & $\begin{array}{l}\text { Mesure le degré de consensus } \\
\text { entre les interviewés sur les } \\
\text { parties de plante utilisées. }\end{array}$ \\
\hline
\end{tabular}

Sources : Byg et Baslev (2001), Gomez-Beloz (2002), Koura et al. (2011).

\section{Résultats}

\section{Zones de distribution géographique de G. kola dans les communes d'étude}

La figure 2 présente les différentes zones de distribution géographique de G. kola dans les communes d'étude. Les points noirs présents sur la figure montrent les points d'occurrences enregistrés de G. kola dans les trois communes. A l'unanimité, les propriétaires enquêtés à Avrankou, Adjarra et Ifangni, ont indiqué que G. kola se retrouve principalement dans les jardins de case, les champs et les plantations de palmier à huile. Espèce des milieux humides, elle ne nécessite pas de soins culturaux spécifiques pour donner une production. Elle résiste aux intempéries et aux phénomènes naturels, à part les feux. 


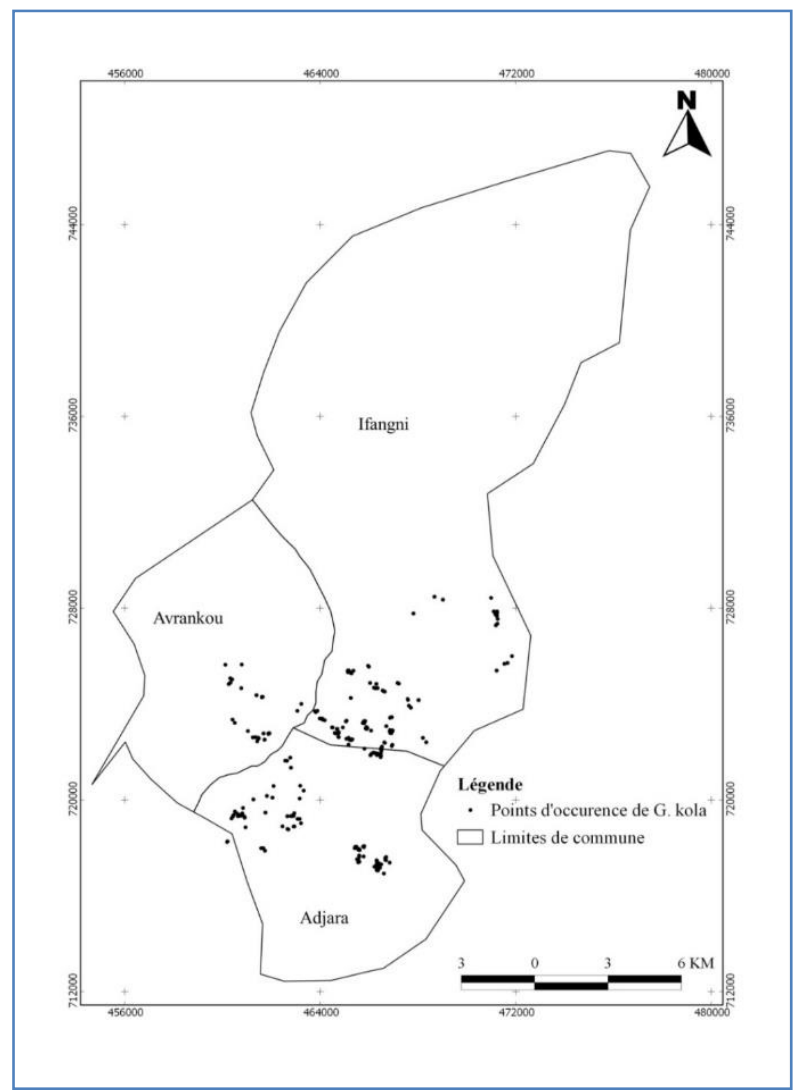

Figure 2 : Carte de distribution géographique de Garcinia kola dans les communes d'étude.

\section{Historique de G. kola dans la zone d'étude}

Pour la majorité des enquêtés $(98,6 \%)$, les pieds de G. kola sont transmis par héritage d'une génération à l'autre. Notons que les femmes n'ont pas la chance d'hériter des pieds de G. kola dans toutes les communes. Les pieds proviendraient de graines pulpées ou de fruits concassés, jetés consciemment ou inconsciemment à la volée, et ayant germé naturellement. L'espèce se retrouve pour la plupart du temps dans les jardins de case $(78,26$ $\%)$, les plantations de palmier à huile $(19,13 \%)$ et les jachères $\quad(2,61 \%)$ parcourus. Depuis de nombreuses décennies jusqu'à l'époque contemporaine, les populations ont manifesté un intérêt pour G. kola. En effet, les pieds rencontrés ont un âge estimé entre 10 et environ 400 ans selon les informations apportées par les propriétaires enquêtés. Les pieds les plus âgés (d'âge estimé à 400 ans) ont été rencontrés à Avrankou et à Adjarra [selon $70 \%$ des propriétaires enquêtés dans la commune d'Avrankou et $60 \%$ de ceux de la commune d'Adjarra]. A Ifangni, les dires de l'ensemble des enquêtés permettent de situer le début des plantations vers 1913. Les plus jeunes des pieds âgés de 10 ans (mise en place vers 2003) ont été rencontrées dans la commune d'Avrankou. 
Le nombre de pieds de $G$. kola dans la région d'étude a progressivement baissé au cours du temps, sous l'influence de différents facteurs. Ainsi, à partir de 1900, il y a eu une forte régression de l'espèce résultant de la réticence de la population $(98,6 \%)$ quant à sa plantation. Les raisons de cette réticence des populations sont :

- la longue durée ( 8 ans) entre la plantation et la première fructification de l'arbre ;

- les préjugés liés à sa plantation : «quand on plante, on meurt avant la première fructification », «c'est un refuge des hiboux (oiseaux associés à la sorcellerie) »;

- la plantation est réservée aux vieux.

Par ailleurs, pour l'ensemble des enquêtés, les pressions anthropiques (construction des logements, construction des routes, cueillette abusive des fruits, coupe incontrôlée du bois et autres parties de l'espèce pour divers usages, etc.) constituent aussi des facteurs qui sont à l'origine de cette régression de l'espèce dans le milieu d'étude.

\section{Retombées financières générées par les graines de $\boldsymbol{G}$. kola}

Les moyennes du nombre de pieds de G. kola plantés par propriétaire, de la recette annuelle par pied et de la recette totale annuelle par propriétaire sont présentées par localité au tableau 2. Les valeurs de recettes moyennes obtenues par pied par an et les recettes totales par propriétaire par an dans la commune d'Adjarra, sont significativement supérieures $(\mathrm{p}<0,001)$ aux valeurs obtenues par an dans les deux autres communes. Les plus faibles valeurs de recettes moyennes par pied par an et de recettes totales par propriétaire par an sont enregistrées dans la commune d'Ifangni à cause de la non proximité des points de vente (marchés) des graines. Les propriétaires d'Ifangni n'aiment pas se déplacer vers les marchés pour la vente de leurs graines à cause du coût de transport qu'ils estiment un peu cher.

Les pieds de G. kola sont mises en place pour subvenir aux besoins immédiats de la famille et de la collectivité. En effet, l'arbre constitue un fonds de garantie et est utilisé aussi comme gage selon $90 \%$ des propriétaires d'Avrankou. Les graines sont commercialisées par tous les enquêtés. Les pieds procurent des revenus car les recettes issues de la vente des graines (1036000 FCFA/propriétaire/an à Adjarra, 356250 FCFA/propriétaire/an à Avrankou et 290892 FCFA/propriétaire/an à Ifangni) constituent une assurance pour la vie. Ces retombées financières sont obtenues moyennant peu d'entretiens. L'usage d'engrais chimiques n'est pas indispensable et seules les coques du fruit sont utilisées comme fertilisants. Selon les dires des enquêtés, G. kola est semblable au palmier à huile (Elaeis guineensis) en termes d'importance socio-économique. Les propriétaires disent que presque toutes les parties de 
l'arbre sont utilisées dans divers domaines. L'espèce génère des profits sur une longue période.

Tableau 2 : Nombre moyen de pieds de Garcinia kola, recette moyenne annuelle par pied et recette totale annuelle par planteur selon les localités.

\begin{tabular}{|c|c|c|c|}
\hline $\begin{array}{c}\text { Comm } \\
\text { une }\end{array}$ & $\begin{array}{c}\text { Nombre moyen de } \\
\text { pieds/propriétaire }\end{array}$ & $\begin{array}{c}\text { Recette } \\
\text { moyenne/pied/an } \\
\text { (FCFA) }\end{array}$ & $\begin{array}{c}\text { Recette } \\
\text { totale/propriétaire/an } \\
\text { (FCFA) }\end{array}$ \\
\hline $\begin{array}{c}\text { Adjarr } \\
\text { a }\end{array}$ & 4 & $259000 \mathrm{a}$ & $1036000 \mathrm{a}^{* * *}$ \\
\hline $\begin{array}{c}\text { Avrank } \\
\text { ou }\end{array}$ & 3 & $118750 \mathrm{~b}$ & $356250 \mathrm{~b}^{* * *}$ \\
\hline Ifangni & 3 & $96964 \mathrm{~b}^{* * *}$ & $290892 \mathrm{~b}^{* * *}$ \\
\hline
\end{tabular}

N.B. : Les valeurs suivies de diverses lettres dans une même colonne sont significativement différentes au seuil de $5 \%$ (ANOVA, test de Newman-Keuls).

Caractérisation des savoirs des populations locales et leurs relations avec G. kola

\section{Diversité et répartition de la connaissance des interviewés}

Toutes les personnes interviewées au cours de l'enquête utilisent $G$. kola. Les valeurs de l'indice de diversité des interviewés (ID) et de l'indice d'équitabilité des interviewés (IE) varie de 0,20 à 0,93 . Les hommes de façon globale détiennent le plus de connaissances sur l'espèce (ID $=0,93$; $\quad$ IE $=0,93$ ) que les femmes (ID =0,48; IE =0,48). Les hommes d'âge supérieur à 40 ans détiennent le plus de connaissances (ID $=0,91 ; \mathrm{IE}=0,91)$ suivis des hommes d'âge inférieur à 40 ans (ID $=0,64$; IE $=0,64$ ). Les femmes qui détiennent le plus de connaissances sur G. kola sont les vieilles femmes (ID $=0,46$ et IE = 46) (Tableau 3). Malgré ces écarts, il n'y a pas de différence significative entre les valeurs. Garcinia kola est une espèce bien connue des populations dans les différentes localités couvertes par l'étude. A cet effet, la dénomination de l'espèce par la population est «Ahowétin » qui serait d'origine fon.

Tableau 3 : Evaluation des connaissances sur G. kola suivant les catégories d'âge et de sexe.

$$
\text { Nombre total d'enquêtés }
$$

Nombre d'usages spécifiques cités

Nombre de sous-catégories d'usages

$$
\text { cités }
$$

Nombre de catégories d'usages cités

6

\begin{tabular}{ccc}
\cline { 2 - 3 } & ID (Ecart-type) & IE (Ecart-type) \\
\hline Total ID & $0,91(0,87) \mathrm{a}$ & $0,91(0,87) \mathrm{a}$ \\
Total pour femmes & $0,48(0,42) \mathrm{a}$ & $0,48(0,42) \mathrm{a}$ \\
Femmes $<40$ & $0,20(0,35) \mathrm{a}$ & $0,20(0,35) \mathrm{a}$ \\
Femmes $\geq 40$ & $0,46(0,40) \mathrm{a}$ & $0,46(0,40) \mathrm{a}$ \\
\hline
\end{tabular}




\begin{tabular}{ccc}
\hline Total pour hommes & $0,93(0,91) \mathrm{a}$ & $0,93(0,91) \mathrm{a}$ \\
Hommes $<40$ & $0,65(0,87) \mathrm{a}$ & $0,65(0,87) \mathrm{a}$ \\
Homme $\geq 40$ & $0,91(0,87) \mathrm{a}$ & $0,91(0,87) \mathrm{a}$ \\
Niveau de signification $(\mathrm{p})$ & 0,055 & 0,055 \\
\hline
\end{tabular}

Pour chaque paramètre, les moyennes suivies des mêmes lettres ne sont pas significativement différents (test de Student-Newman-Keuls)

\section{Catégories, sous-catégories d'usages et usages spécifiques de G. kola}

Six (6) catégories d'usages de G. kola ont été recensées dans les communes: usages médicinaux, alimentaires (pour les hommes et les animaux), commerciaux, ombrage, bois et usages médico-magiques. Certaines catégories d'usages ont été divisées en sous-catégories d'usages / usages spécifiques. En ce qui concerne la catégorie « usages médicinaux », les souscatégories / usages spécifiques recensés sont: "maladies de l'appareil digestif» (constipation, maux de ventre, vomissement), «maladies stomatologiques» (maladies bucco-dentaires), «maladies de l'appareil respiratoire » (rhume, asthme, toux), « maladies dermatologiques » (maladies de la peau), «maladies infectieuses» (paludisme) et "symptômes et syndromes particuliers » (courbature, fièvre, hoquet, maux de hanche, fatigue). La catégorie « usages alimentaires » comporte deux sous-catégories : l'usage des feuilles de l'espèce comme fourrage pour nourrir les animaux et la consommation des graines par les hommes. En ce qui concerne les usages commerciaux, elles prennent en compte la vente des graines de l'espèce. La catégorie d'usage «bois» comporte trois (3) usages spécifiques: usages comme bois de charpente, usages comme bois de feu et usages comme brosse végétale. La catégorie « usages médico-magiques » comportent deux souscatégories : « usages pour se protéger contre les serpents » et « usages en tant que parole agissante $»$.

C'est la catégorie « usages médicinaux » qui a enregistré la plus grande valeur de diversité des usages ( $\mathrm{UD}=0,64$ à Adjarra, $\mathrm{UD}=0,61$ à Avrankou et $\mathrm{UD}=0,68$ à Ifangni) (Tableau 4). Tout ceci fait de cette catégorie d'usage, celle non seulement la plus répandue mais aussi la plus diversifiée.

Il ressort de la présente étude que les usages médicinaux, «alimentaires (pour l'alimentation des hommes)» et commerciaux sont largement les plus répandus avec respectivement 19,90\%, 19,90\% et $19,90 \%$ des cas rencontrés, suivi des usages « bois » $(18,91 \%)$, « ombrage » $(18,91 \%)$, usages pour l'alimentation des animaux $(2,32 \%)$ et usages médicomagiques $(0,17 \%)$. 
Tableau 4 : Valeur de diversité des usages (UD) et valeur d'équitabilité des usages (UE).

\begin{tabular}{|c|c|c|c|c|c|c|}
\hline \multirow{2}{*}{ Catégories d'usages } & \multicolumn{2}{|c|}{ Adjarra } & \multicolumn{2}{c|}{ Avrankou } & \multicolumn{2}{c|}{ Ifangni } \\
\cline { 2 - 7 } & UD & UE & UD & UE & UD & UE \\
\hline Usages médicinaux & 0,64 & 1,00 & 0,61 & 1,00 & 0,68 & 1,00 \\
\hline Usages alimentaires & 0,09 & 0,14 & 0,09 & 0,14 & 0,05 & 0,08 \\
\hline Ombrage & 0,05 & 0,07 & 0,04 & 0,07 & 0,05 & 0,08 \\
\hline Bois & 0,14 & 0,21 & 0,13 & 0,21 & 0,16 & 0,23 \\
\hline Usages médico-magiques & 0,05 & 0,07 & 0,09 & 0,14 & 0,00 & 0,00 \\
\hline Usages commerciaux & 0,05 & 0,07 & 0,04 & 0,07 & 0,05 & 0,08 \\
\hline
\end{tabular}

\section{Parties utilisées de $G$. kola dans la zone d'étude}

Les parties de $G$. kola utilisées sont les graines $(65,02 \%), \%)$, l'écorce $(13,90 \%)$, les racines $(13,01 \%)$, les tiges $(3,14 \%)$ les fruits $(3,14 \%)$ et les feuilles $(1,79 \%)$. Les graines, l'écorce et les racines sont les parties les plus utilisées.

Le tableau 5 présente les valeurs consensuelles des parties de G. kola utilisées dans les différentes communes. Il faut noter que les enquêtés rencontrés sont tous du groupe socio-linguistique Goun. L'analyse des valeurs consensuelles des parties de G. kola s'est alors basée sur les valeurs obtenues par commune. Il ressort de cette analyse que les graines sont les organes les plus utilisés quelle que soit la commune considérée. Cela pourrait s'expliquer par les multiples usages (médicinaux, alimentaires, commerciaux, médicomagiques) qui sont faites de ces graines.

Tableau 5 : Valeurs consensuelles des parties de G. kola.

\begin{tabular}{|c|c|c|c|c|c|c|c|c|c|c|c|c|c|c|}
\cline { 2 - 15 } \multicolumn{1}{c|}{} & \multicolumn{1}{c|}{ Adjarra } & \multicolumn{1}{c|}{ Avrankou } & \multicolumn{5}{c|}{ Ifangni } \\
\cline { 2 - 8 } & Arr2 & Arr2 & Adj1 & Adj2 & Az & De & Mal & Sad & Avra & Dj & Aka & Arr1 & D_wo & Agb \\
\hline Graines & 1,00 & 1,00 & 0,91 & 1,00 & 1,00 & 1,00 & 0,59 & 1,00 & 0,33 & 0,40 & 0,71 & 0,53 & 0,84 & 1,00 \\
\hline Fruits & 0,00 & 0,00 & 0,00 & 0,00 & 0,00 & 0,00 & 0,00 & 0,00 & 0,00 & 0,00 & 0,00 & 0,00 & 0,00 & 0,00 \\
\hline Feuilles & 0,00 & 0,00 & 0,00 & 0,00 & 0,00 & 0,00 & 0,00 & 0,00 & 0,00 & 0,00 & 0,00 & 0,00 & 0,00 & 0,00 \\
\hline Racines & 0,00 & 0,00 & 0,04 & 0,00 & 0,00 & 0,00 & 0,23 & 0,00 & 0,17 & 0,20 & 0,14 & 0,23 & 0,07 & 0,00 \\
\hline Ecorce & 0,00 & 0,00 & 0,04 & 0,00 & 0,00 & 0,00 & 0,18 & 0,00 & 0,33 & 0,30 & 0,14 & 0,23 & 0,07 & 0,00 \\
\hline Tige & 0,00 & 0,00 & 0,00 & 0,00 & 0,00 & 0,00 & 0,00 & 0,00 & 0,17 & 0,10 & 0,00 & 0,00 & 0,00 & 0,00 \\
\hline
\end{tabular}

Les résultats d'Analyse en Composantes Principales (ACP) appliquée aux valeurs consensuelles des parties de la plante (CPP) montrent que les deux premiers axes expliquent $90,67 \%$ des variations observées. A cet effet, ces axes sont retenus pour expliquer la relation existant entre les arrondissements (premiers niveaux de subdivision administrative des communes) et les valeurs consensuelles des parties de G. kola utilisées. Le tableau 6 montre les coefficients de corrélation entre les parties de G. kola et les deux axes. Le 
premier axe montre une corrélation positive avec les racines, l'écorce et les tiges et est corrélé négativement avec les graines tandis que le second axe montre une corrélation positive avec les feuilles et les fruits.

Tableau 6 : Corrélation entre les parties de G. kola et les axes d'ACP.

\begin{tabular}{|c|c|c|}
\cline { 2 - 3 } \multicolumn{1}{c|}{} & Dim.1 & Dim.2 \\
\hline Graines & $-0,98$ & 0,15 \\
\hline Fruits & 0,42 & 0,91 \\
\hline Feuilles & 0,42 & 0,91 \\
\hline Racines & 0,88 & $-0,18$ \\
\hline Ecorce & 0,94 & $-0,32$ \\
\hline Tiges & 0,77 & $-0,18$ \\
\hline
\end{tabular}

La projection des parties (graines, tiges, racines, fruits, feuilles et écorce) et des arrondissements dans ce système d'axes (Figure 3) révèle que les graines de $G$. kola sont plus utilisées dans la commune d'Adjarra, dans les arrondissements ci-après : $2^{\text {ème }}$ arrondissement, $4^{\text {ème }}$ arrondissement, Adjarra1, Adjarra2, Azowlissè, Mèdédjonou et Dèmè. Les tiges, racines et écorce sont plus utilisés par les populations des arrondissements d'Avrankou et de Djomon dans la commune d'Avrankou, de l'arrondissement d'Akadja dans la commune d'Ifangni et enfin de l'arrondissement de Malanhoui dans la commune d'Adjarra. Les fruits et les feuilles, quant à eux, sont plus utilisés par les populations de l'arrondissement de Sado dans la commune d'Avrankou.

Variables factor map (PCA)
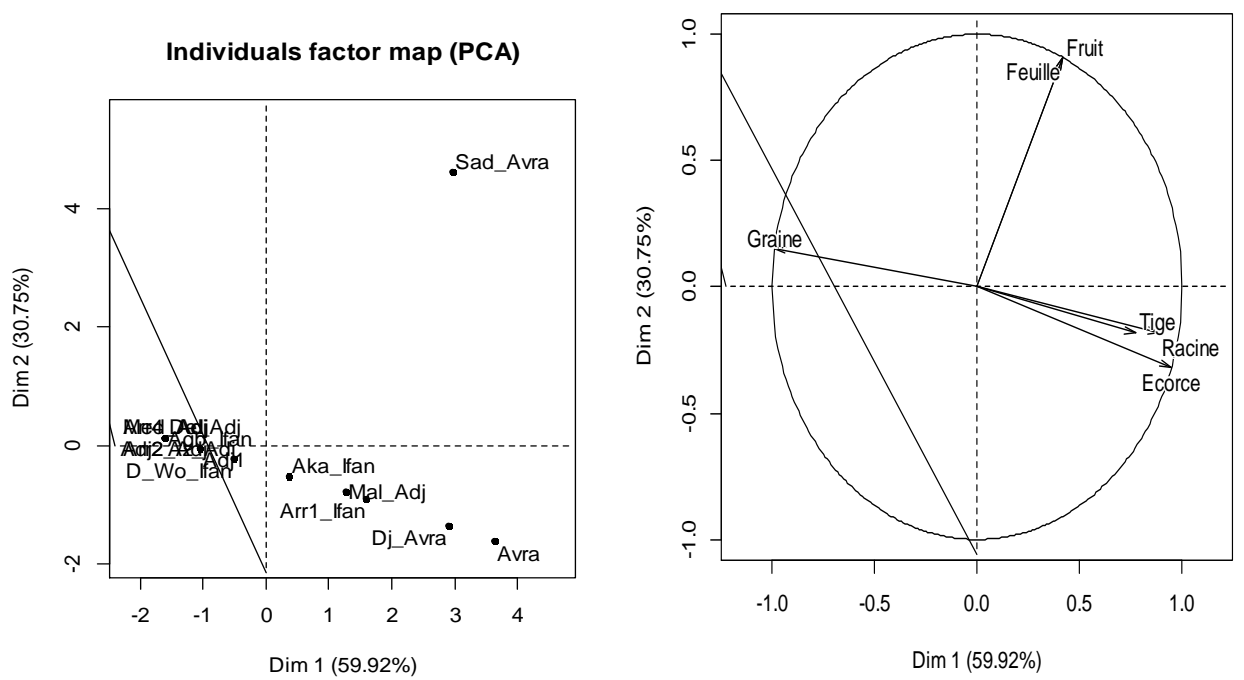

Figure 3 : Projection des groupes ethniques sur le système d'axes défini par les différents organes.

N.B. : Arr2_Adj $=2^{\text {ème }}$ arrondissement d'Adjarra ; Arr4_Adj $=4^{\text {ème }}$ arrondissement 
d'Adjarra $;$ Adj1 = Adjarra1 $;$ Adj2 =Adjarra2 $;$ Az_Adj = Azowlissè $;$ De_Adj $=$ Demè d'Adjarra $;$ Mal_Adj $=$ Malanhoui d'Adjarra $;$ Med_Adj= Mèdédjonou $;$ Sad_Avra $=$ Sado

d'Avrankou ; Avra = Avrankou ; Dj_Avra = Djomon d'Avrankou ; Aka_Ifan=Akadja ;

D_Wo_Ifan $=$ Damè-wogon d'Ifangni ; Arr1_Ifan $=1^{\text {er }}$ arrondissement d'Ifangni ; Agb_Ifan = Aglangandan .

\section{Discussion}

\section{Historique de Garcinia kola et son insertion dans les systèmes de production}

Cette étude a permis d'identifier les principales formations où se retrouve Garcinia kola dans les communes d'Avrankou, Adjarra et Ifangni. Ce sont : les jardins de case, les champs et les plantations de palmier à huile. L'absence de l'espèce dans les formations naturelles confirme les résultats de (Adomou et al., 2011). L'étude révèle aussi la régression de Garcinia kola au cours du temps. Cette situation découle de la destruction progressive de ses habitats par les actions anthropiques (construction des routes, utilisation du tronc dans la fabrication des charpentes, utilisation des branches comme bois de feu, etc.). D'après Anegbeh et al. (2006), au Nigéria, le déboisement rapide et l'exploitation des forêts naturelles ont affecté sérieusement les populations de l'espèce d'où la faible disponibilité des graines dans les marchés. Pour le moment, la plantation de l'espèce est limitée et ne permet pas de compenser cette tendance régressive. Les handicaps à la plantation sont : les préjugés sur la longue durée avant la première fructification et la nécessité de traiter les graines pour un meilleur taux de germination. En ce qui concerne les préjugés sur la longue durée avant la première fructification, selon certains enquêtés, celui qui plante cet arbre, meurt avant la première fructification donc ne jouit pas des fruits de l'arbre. Les premiers fruits apparaissent sur l'arbre vers l'âge de 7 ans selon certains propriétaires.

\section{Importance socio-économique et usages de Garcinia kola Contribution de l'espèce à l'économie des propriétaires}

Garcinia kola représente une source non négligeable de revenus pour les propriétaires. Les revenus sont plus élevés dans la commune d'Adjarra, car cette localité abrite un marché d'échange des graines de l'espèce. De plus, le nombre moyen de pieds par propriétaire est plus élevé dans la commune d'Adjarra que dans les deux autres communes. Le marché d'Adjarra constitue un grand carrefour de la vente des graines de G. kola. Par conséquent, le prix des graines est élevé dans cette commune par rapport aux autres communes. Les communes d'Avrankou et d'Ifangni sont des communes voisines de celle d'Adjarra du point de vue géographique. Les faibles recettes obtenues dans les communes d'Avrankou et d'Ifangni résident également dans le fait que la plupart du temps, les propriétaires de ces communes ne vont pas vendre les graines dans le marché d'Adjarra. Les échanges se font le plus souvent à la 
maison.

Un commerce intensif se fait dans les communes d'Adjarra, d'Avrankou et d'Ifangni à cause de l'abondance de l'espèce et de la proximité du Nigéria où la demande des graines se fait sentir. Au Nigéria, la demande des graines de $G$. kola est actuellement forte alors que l'offre locale est faible (Anegbeh et al., 2006). L'espèce est très prisée par la population et est même recherchée préférentiellement, pour l'amélioration des conditions de vie des propriétaires. Cette préférence est surtout motivée par sa valeur commerciale qui contribue significativement à l'économie des exploitants ou des utilisateurs. N'guessan et al. (2012) affirment que la vente des graines de $G$. kola apporte des revenus non négligeables aux populations des sites de production et de commercialisation d'amandes d'Elibou en Côte d'Ivoire. De plus, la valeur marchande de la graine de G. kola, en général de 1000 FCFA à 1500 FCFA le kilogramme, lui accorde une importance similaire au cacao qui était de $1000 \mathrm{FCFA} / \mathrm{kg}$ en 2014 (Aké, 2015).

\section{Connaissances ethnobotaniques sur l'espèce}

La connaissance de l'utilisation endogène des ressources naturelles est essentielle à l'élaboration des stratégies de conservation (Achigan-Dako et al., 2011). G. kola est très apprécié en raison des multiples usages faits de ses organes (feuilles, fruits, graines, écorce, racine et tige) (Agyili et al., 2006). Les résultats de la présente étude ont montré que les graines de G. kola sont plus utilisées tant sur le plan alimentaire que sur les plans médicinal, médicomagique et commercial. Eyog Matig et al. (2006) avaient déjà rapporté que les parties les plus utilisées de G. kola au Cameroun sont les graines. De plus, les graines de $G$. kola contiennent d'éléments nutritionnels précieux qui seraient la raison principale de son utilisation dans l'alimentation (Afolabi et al., 2006). La composition du fruit montre qu'il est un bon aliment fournissant des sels biogéniques d'une grande qualité nutritionnelle et pourraient également jouer un rôle dans la prévention et le traitement de certaines pathologies liées à la carence en zinc, en magnésium et en cuivre (Morabandza, 2013). Par ailleurs, les propriétaires de G. kola confèrent à l'essence surtout la graine, d'excellentes qualités médico-magiques et alimentaires qui aideraient les populations consommatrices à détenir des pouvoirs surnaturels qui leur permettraient de se protéger. Le grand nombre d'usages rapportés serait dû à la fréquence relativement élevée de l'espèce dans les communes. En effet, plus une espèce est abondante dans une zone, plus les populations la connaissent et l'utilisent (Albuquerque et al., 2006). Les tiges sont utilisées comme brosse végétale dans la commune d'Avrankou. Les rameaux de $G$. kola sont principalement utilisés comme cure-dents et préviendraient les caries dentaires (Guedje et Fankap, 2001 ; Ballet et al., 2012). 
D'après nos résultats, les hommes âgés de plus de 40 ans détiennent plus de connaissances sur l'utilisation des différentes parties de l'espèce. Six catégories d'usage ont été identifiées à savoir : médicinale, médico-magique, alimentaire, ombrage, commerciale et bois. Les populations utilisent, en effet le bois de G. kola comme bois de service dans la construction des habitations et aussi comme bois de feu. Tous ses organes sont utilisés par les enquêtés pour le traitement de plusieurs maladies/symptômes au nombre desquels : constipation, maux de ventre, vomissement, maladies bucco-dentaires, rhume, asthme, toux, maladies de la peau, paludisme, courbature, fièvre, hoquet, maux de hanche, fatigue. A cet effet, Oguntola (2008) explique que c'est une " plante miracle » parce que presque toutes ses parties ont été jugées d'une importance médicinale avérée. Guedje et Fankap (2001) ont aussi rapporté que l'écorce et la tige sont couramment utilisées pour accélérer la fermentation de la sève du palmier à huile ou du palmier raphia.

\section{Implications pour la conservation de G. kola}

La principale menace sur les pieds de G. kola semble être la commercialisation de plus en plus accrue des graines pour divers usages. L'espèce est consommée dans tous les départements du Bénin à raison d'environ $200 \mathrm{~g}$ de graines par habitant et par an (Eyog Matig et al., 2006). Les localités d'Adjarra, d'Avrankou et d'Ifangni se sont révélées les derniers refuges pour l'espèce et mérite une attention particulière de la part des conservateurs. A ce propos, la mise en place d'un parc à G. kola dans ces différentes communes permettra de sauvegarder l'espèce in situ. Par ailleurs, les populations devraient être régulièrement sensibilisées sur l'importance de préserver l'espèce dont l'extinction affectera non seulement la biodiversité nationale mais aussi les valeurs socio-culturelles qui lui sont liées.

Néanmoins plusieurs pratiques endogènes permettent encore de sauvegarder l'espèce dans les environs immédiats des maisons.

Les principales stratégies de conservation de G. kola comprennent :

(1) la préservation des pieds dans les systèmes de culture à base d'arbres tels que les jardins de case, les plantations de palmier à huile et les champs ;

(2) le défrichement sélectif et la protection de la repousse dans les plantations ;

(3) le développement des pépinières de G. kola et les efforts de plantations dans les jardins botaniques et chez les planteurs privés.

Il faudra approfondir et développer les pratiques qui permettent une meilleure propagation de l'espèce. Des stratégies de propagation (par graine, par bouturage, etc.) doivent être mises en œuvre au profit des populations des communes d'Adjarra, d'Ifangni et d'Avrankou notamment celles qui utilisent et commercialisent l'espèce. Des essais de plantations devront être faits dans les domaines classés de l'état pour assurer la conservation in situ de l'espèce. 


\section{Conclusion}

La présente étude a montré que l'espèce ne se trouverait plus à l'état naturel mais en plantation dans la zone d'étude. Les utilisations que les populations d'Avrankou, d'Adjarra et d'Ifangni font des organes de G. kola, une espèce connue des populations du sud-est du Bénin sont principalement alimentaires, médicinales, ombrage, bois, médico-magiques et commerciales. Aussi, l'espèce représente-t-elle une source non négligeable de revenus pour les propriétaires des pieds de G. kola.

Des études approfondies doivent être faites sur la commercialisation des divers organes dans les marchés sur le plan national. Aussi, des études doivent-elles être réalisées sur 1'exploitation des graines afin de prendre des décisions efficaces et efficientes pour la gestion durable de l'espèce.de plus il est important de connaitre la production fruitière de l'espèce.

Des études doivent être menées sur les compositions biochimiques des organes de G. kola suivant leurs provenances. Ceci pourrait aider à rechercher et expliquer 1'effet des provenances et des caractéristiques physicochimiques des sols sur la valeur nutritionnelle des graines de l'espèce.

Des recherches doivent aussi être faites sur la variabilité morphologique existante au niveau des fruits de $G$. kola suivant différentes zones au Bénin. Cela sera un paramètre utile à une amélioration génétique de l'espèce répondant aux besoins et aux moyens des populations rurales.

Des recherches doivent aller dans le sens de la réintroduction de l'espèce dans les différentes formations végétales au Bénin.

Enfin, des études doivent être faites afin de promouvoir la domestication pour augmenter l'utilisation durable de G. kola. Cela pourrait aussi aider à améliorer les revenus et augmenter les pouvoirs d'achat des populations au niveau local.

\section{References:}

1. Achigan-Dako, E., N'Danikou, S., Assogba-Komlan, F., Ambrose-Oji, B., Ahanchede, A., Pasquini, M. (2011). Diversity, Geographical, and Consumption Patterns of Traditional Vegetables in Sociolinguistic Communities in Benin: Implications for Domestication and Utilization. Economic Botany, 65:129-145.

2. Adomou, A. C., Agbani, O. P. \& Sinsin, B. (2011). Plantes. In Protection de la Nature en Afrique de l'Ouest: Une Liste Rouge pour le Bénin. Nature Conservation in West Africa: Red List for Benin, Neuenschwander P, Sinsin B, Goergen G (eds). International Institute of Tropical Agriculture: Ibadan, Nigeria; 21-46.

3. Adjanohoun, E. A., Adjakidjè, V., Ahyi, M. R., Aké, A.L., Akoègninou, A., d'Almeida, J., Apovo, F., Boukef, K., Chadaré, M., Cusset G., Dramane, K., Yme, J., Gassita, J. N., Gbaguidi, N., Goudoté, E., 
Guinko, S., Houngnon, P., Issa, L., Keita, A., Kiniffo, H.V., KoneBamba, D., Musampa, N. A., Saadou, M., Sodogandji, T., de Souza, S., Tchabi, A., Zinsou, D. C. \& Zohoun, T. (1989). Contribution aux études ethnobotaniques et floristiques en République Populaire du Bénin., Paris, Edition de l'ACCT, 895 p.

4. Afolabi, F. E., Eleyinmi, D. C., Bressler, D. C., Isiaka, A., Amoo, I. A., Peter Sporns, P., Aladesanmi, A., Oshodi, A. A., 2006. Chemical composition of bitter cola (Garcinia kola) seed and hulls. POLISH journal of food and nutrition sciences. Vol. 15/56, No 4, pp. 395-400.

5. Agyili, J., Sacande, M. \& Kouame, C. (2006). Garcinia kola Heckel. Seed Leaflet, No. 113.

6. Akerele, O.S. Obasuyi, O. Ebomoyi, M.I., Oboki, I.E. and Umumarongie,O.H. (2007). Antimicrobial activity of the ethanol extract and Fractions of the seeds of Garcinia kola Heckel (Guttiferae). Afr. J. of Biotechnol. Vo1.7(2), Pp. 169-172

7. Aké, C. B. (2015). Etude ethnobotanique des plantes et des champignons spontanés, utilisées en alimentation dans le Département d'Agboville et le District d'Abidjan (Côte d'Ivoire). Thèse de Doctorat de l'Université Félix Houphouët Boigny de Cocody-Abidjan (Côte d'Ivoire), UFR Biosciences, 187 p.

8. Akoègninou, A., Vander Burg, W. J., Vander, L. J. G. \& Maesen. (2006). Flore Analytique du Bénin. Backhuys publisher Wageningen, p. 476.

9. Albuquerque, U. P., Lucena, R. F. P., Monteiro, J. M., Florentino, A. T. N. \& Almeida, C. F. C. B. R. (2006). Evaluating two quantitative ethnobotanical techniques. Ethnobotany research and applications, 4:51-60.

10. Ambé, G. A. (2001). Les fruits sauvages comestibles des savanes guinéennes de Côte-d'Ivoire : état de la connaissance par une population locale, les Malinké. Biotechnol. Agron. Soc. Environ. 5 (1) : 43-58.

11. Anegbeh, P. O., Iruka, C. \& Nkirika, C. (2006). Enhancing Germination Of Bitter Cola (Garcinia Kola) Heckel: Prospects For Agroforestry Farmers In The Niger Delta p. 8.

12. Awokou, K. S., Ganglo, C. J., Azontondé, H., Adjakidjè, V. \& De foucault B. (2009). Caractéristiques structurales et écologiques des phytocénoses forestières de la forêt classée d'Itchèdè (Département du plateau, Sud-Est Bénin). Sciences \& Nature 6(2) : 125-138.

13. Ballet, P., Kouamé, N .M. T., Aké, C. B. (2012). Inventaire des produits forestiers non ligneux à valeur marchande en vue de leur domestication et valorisation, Rapport final de projet, ONG Côte d'Ivoire Ecologie, $57 \mathrm{p}$. 
14. Byg, A. \& Balslev, H. (2001). Diversity and use of palms in Zahamena, eastern Madagascar. Biodiversity and Conservation 10: 951-970.

15. CTA. (2007). Les Fruitiers sauvages d'Afrique. p. 10-41.

16. Eyog Matig, Ndoye, O., Kengue, J. \& Awono, A. (2006). Les fruitiers forestiers comestibles du Cameroun. CIFOR. Cameroun. IPGRI, 204 p.

17. FAO. (2011). Evaluation des Ressources Forestières Mondiales. Rome, Italie. 320 p.

18. Farombi, E. O., Tahnteng, J. G., Agboola, A. O., Nwankwo, J. O. \& Emerole, G. O. (2000). Chemoprevention of 2- acetylaminofluorene induced hepatotoxicity and lipid peroxidation in rats by kolaviron a Garcinia kola seed extract. Food Chem. Toxicol., 38(6):535-41.

19. Gomez-Beloz, A. (2002). Plant use knowledge of the Winikina Warao: the case for questionnaires in ethnobotany. Economic Botanic, (pp. 231-241).

20. Guedje, N. M. \& Fankap, R. (2001). Utilisations traditionnelles de Garcinia lucida et Garcinia kola (Clusiaceae) au Cameroun. National Botanic Garden Of Belgium. 71: 747-758.

21. Han, Q. B., Lee S. F., Qiao, C. F., He Z. D., Song, J. Z., Sun, H. D. \& $\mathrm{Xu}, \mathrm{H} . \mathrm{X}$. (2005). Complete NMR assignments of the antibacterial biflavonoid GB1 from Garcinia kola. Chem. Pharm. Bull. 53(8): 10341036.

22. Kabuye, C. (2000). Généralités sur la recherche économique et les Produits Forestiers Non Ligneux. FAO. Rome, (pp 117-121).

23. Koura, K., Ganglo, J. C., Assogbadjo, A. E., \& Agbangla, C. (2011). Ethnic differences in use values and use patterns of Parkia biglobosa in Northern Benin. Journal of Ethnobiology and Ethnomedicine. 7:42

24. Kouchadé, M. (2013). Connaissances endogènes et évaluation écophénotypique de Garcinia kola (heckel) au sud-Bénin. 66 p.

25. Mac Arthur, R. H., Wilson, E. O. (1967). The theory of Island Biogeography. Princeton University Press, New Jersey, 224 p.

26. Monteiro, J. M., Albuquerque, U. P., Lins Neto, E. M. F., Araùjo, E. L. \& Amorim, E. L. C. (2006). Use Patterns and Knowledge of Medicinal Species among Two Rural Communities in Brazil's Semi-Arid Northeast-ern Region. Journal of Ethnopharmacology, 105:173-186.

27. Morabandza, C. J., Ongoka, R. P., Matini, L., Epa, C., Nkounkou, L.C. \&, Abena, A.A. (2013). Chemical Composition of the Mesocarp of Garcinia kola Heckel (Clusiaceae). Fruit Research Journal of Recent Sciences 2(1):53-58.

28. Neuenschwander, P., Sinsin B. \& Goergen, G. (eds). (2011). Protection de la Nature en Afrique de l'Ouest : une liste rouge pour le Bénin. 
International Institute of Tropical Agriculture, Ibadan, Nigéria. 365 p. 29. N'guessan, K. A., Koffi, E., Gnahoua, G. M., Coulibaly, B. \& Tahouo, O. (2012). Le système de production et de commercialisation des plants pour sauver l'arbre du «petit cola» en Côte d'Ivoire CNRA ISBN 978-2-917074-10-7.

30. Oguntola, S. (2008). How bitter kola can improve your health. Niger. Tribun Health. Ng/030/2008 LH2. LFML.

31. Onunkwo, G. C., Egeonu, H. C., Adikwu, M. U., Ojile, J. E. \& Olowosulu, A.K. (2004). Some physical properties of appendixtted seed of Garcinia kola (Heckel). Chem Pharm Bull, 52(6):649-53.

32. Sibanda, T. (2007). Assessment Of Anti-Bacterial Potentials Of Garcinia Kola Seed Extracts And Their Interactions With Antibiotics. Department Of Biochemistry And Microbiology, Faculty Of Science And Agriculture University of Fort Hare, Alice. P 12-133.

33. Taiwo, O., Xu H. X. \& Lee, S. E. (1999). Antibacterial activities of extracts from Nigerian chewing sticks. Phytoter Res. 13(8): 675-679.

34. Tchoundieu, Z., Duguma, B., Tiencheu, M-L, et Ngo-Mpeck, M.L. (2000). La domestication des arbres indigènes agroforestiers : la stratégie du CIRAF dans les régions tropicales humides d'Afrique Centrale et d'Afrique de l'Ouest. Les produits forestiers non ligneux en Afrique Centrale: Recherche actuelles et perspectives pour la conservation et le développement. Edité par T. C. H. Sunderland, L.E. Clark et P. Vantomme, FAO, Rome, 171-180 pp.

35. Yakubu, F. B., Adejoh, O. P, Ogunade, J. O. \& Igboanugo, A. B. I. (2014). Vegetative Propagation of Garcinia kola (Heckel). World Journal of Agricultural Sciences. 10 (3): 85-90. 